\title{
Prototype of Positioning System for Automatic Motion Control of Underwater Robot
}

\author{
Xiujing Gao ${ }^{1}$, Feifei Zhang ${ }^{1}$, Masanori Ito ${ }^{1}$, \\ Kiyoshi Mishima ${ }^{1}$, Ribun Onodera ${ }^{2}$ \\ 1: Tokyo University of Marine Science and Technology, Japan \\ 2: Polytechnic University, Japan
}

\author{
Naohiro Inagawa ${ }^{3}$ \\ Ikuo Yamamoto ${ }^{4}$ \\ 3: Nippon Bunri University, Japan \\ 4: Nagasaki University, Japan
}

\begin{abstract}
Recently, the needs of underwater robot used for many kinds of underwater work become higher and higher. In order to control the motion of underwater robot automatically, it is an indispensable to measure its position correctly in real time. Conventional systems are based on time difference or phase lag. However, they must use expensive components, such as transponders or atomic clock, and its system becomes complex. Additionally they require a lot of signal-processing time, so they cannot be used for motion control. As a result, there exist no system which can be used for automatic motion control of underwater robot. Since 2008, we have proposed a new positioning system based on sound propagation loss and sensor network. In this system, we set many buoys that install sound reception unit, GPS receiving equipment and sensor network system on the surface of water. We use sound propagation loss to calculate the distance between robot and buoys, and with combining distance and position data for each buoy, we estimate robot position. In former study, we found that we can get higher SN ratio of signal to measure distance with using the sound of multiple frequency, we proposed a new distance measurement method M_SPL. It was introduced on the paper of "Underwater Acoustics Positioning System Based on Propagation Loss and Sensor Network" (OCEANS 2012 KOREA). In this paper, we introduce the design and construction of prototype system for this positioning system as the results of latest study. It contains transmission unit, reception unit and center unit. Transmission unit follows with principle of M_SPL, is set into underwater robot used as sound source. We also complete reception unit to be used to receive sound signal and calculate distance automatically. Finally, center unit receive distance and each buoy position data what are sent from reception unit, position can be calculated by using these data. We confirmed the performance of transmission unit and receiving unit in water-tank, and it showed that both units can be used effectively. Now we are on a final test of this system in open sea for the actual use of surveying sea floor with the underwater robot made by NAGASAKI UNIVERSITY in Japan.
\end{abstract}

Keywords - Prototype System;Sound Propagation Loss;Sensor Network;Underwater Robot; Automatic Motion Control; Multiply Frequency; M_SPL; D_SPL; Mixed Wave;

\section{INTRODUCTION}

In recent years, the research for the oceanic survey or development of oceanic natural resource is actively advanced. The needs of the underwater robots used for those purpose have been becoming higher and higher. For the autonomous underwater robot, positioning system is one of the important subjects to exercise their full performance. The conventional positioning systems in water are based on the time difference or phase lag of transferred sound from source to receiver. They measure the distance between moving object and station where the position has already fixed. When 3 stations are prepared, we can fix 3 dimensional coordinate of object.

However, the systems are expensive and complex, cannot be used for wide use. In 2008, we proposed a new system using sound propagation loss and sensor network, which is constructed by a simple method of having a transducer on robot and a receiver on float. The distance can be calculated with relation between sound propagation loss and distance. Based on this principle, the conventional distance measurement method called D_SPL is using one frequency sound as sound source, and the sound propagation loss is obtained by using of direct wave of received signal. As the received signal contains both "direct wave" and reflected wave, it is difficult to separate direct wave and reflected wave from the received signal in case of long distance, and the measurement accuracy becomes worse. To solve this problem, we proposed a wide range and highly precise measurement method which called M_SPL. M_SPL uses multiple frequency sound as sound source, measures the level of "mixed wave" in the reception signal to calculate sound propagation loss, and distance can be calculated with relation between sound propagation loss and distance. Additionally, it uses many floats composing sensor network to measures position. In former study, we confirmed the availability and performance of M_SPL, and the theory of positioning system was also established.

In this paper, we introduce the design and construction of prototype system for this positioning system as the results of latest study. Because of the originality of M_SPL, it does not exist equipment can be used respond to M_SPL. We develop a new transmission unit what can send composition signal for multiply frequency, and can be used for M_SPL. To correspond that, we also development a reception unit what will be setup into float as a standard station. It can receive signal from reception transducer and can calculate distance in CPU automatically. Reception unit also used to send distance data and its position data from GPS to center station through 
network. On center station, the CPU calculates robot position and sends it to robot for automatic motion control.

\section{POSITIONING SySTEM}

Fig.1 shows the image of positioning system. This system consists of distance measuring unit, position measuring unit and communication unit.

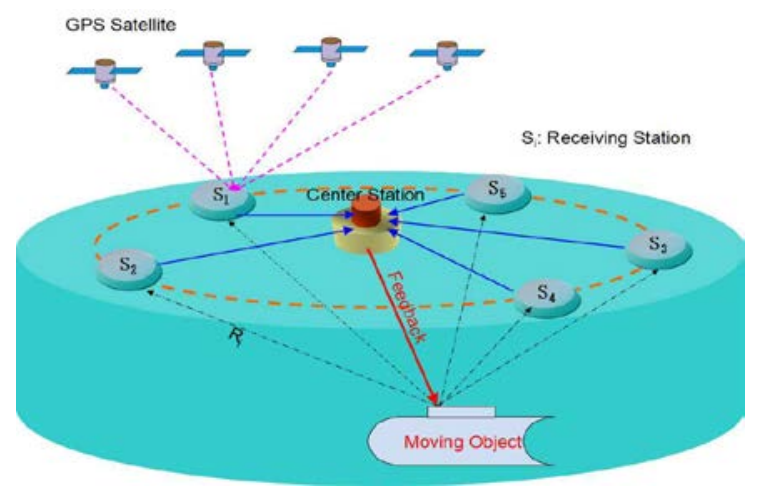

Fig. 1. Image of Positioning System

We use passive sonar in this system. Firstly, distance measuring unit takes charge of distance measurement by CPU in receiving station. Transmission unit is equipped on robot and transmits sound signal continuously. Reception unit receives sound signal and measures the level of it to calculate distance. The position of each receiving station can be measured by GPS unit what is equipped on float. Secondly, the reception unit send distance and float position data to the center station. In center station, center unit calculates robot position and sends data back to robot by communication unit finally.

\section{A. Distance measurement system and method(M_SPL)}

The distance between robot and receiving station is calculated with the principle based on sound propagation loss (SPL). The propagation loss is the attenuation of the sound pressure level according to the distance away from the sound source. The relation between the propagation loss SPL [dB] and the distance $\mathrm{R}[\mathrm{m}]$ can be presented as

$$
S P L=20 \log R+\alpha R+A
$$

Where $a[\mathrm{~dB} / \mathrm{m}]$ is the absorption loss coefficient. There are same empirical equations about measurement for absorption loss coefficient such as "Thorp" equation, "Schulkin and Marsh " equation, "Fisher and Simmons" equation, "Francois and Garrison" equation, "Ainslie and McColm" equation etc. In this study, the absorption loss coefficient is calculated using the most highly accurate from these empirical equations. A is the propagation anomaly, which regards as one of the measurement error factors.

Based on this principle, we proposed the distance measuring system as shown in Fig.2. The parameters can be defined as follows: $\mathrm{V}_{\mathrm{T}}[\mathrm{V}]$, transmitted signal voltage; $\mathrm{V}_{\mathrm{R}}[\mathrm{V}]$, received signal voltage; $T_{g}[d B]$, transmission gain; $R_{g}[d B]$, reception gain; $T_{s}[d B]$, transmission sensitivity; $R_{s}[d B]$, reception sensitivity.

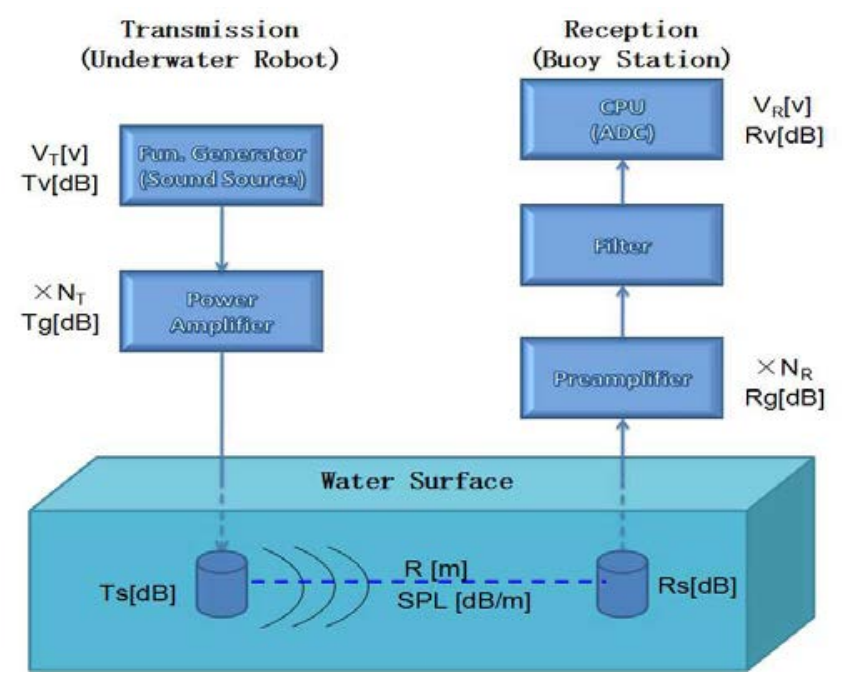

Fig. 2. Distance Measurment System

As a result, sound propagation loss can be calculated as

$$
S P L=20 \log \left(\frac{V_{T}}{V_{R}}\right)+T_{g}+T_{s}+R_{g}+R_{s}
$$

Since the level of transmission signal $V_{T}$ and the parameters of the devices are known, it's clear that distance $\mathrm{R}$ can be calculated if the level of reception signal $V_{R}$ can be measured correctly from equation (1) and (2).

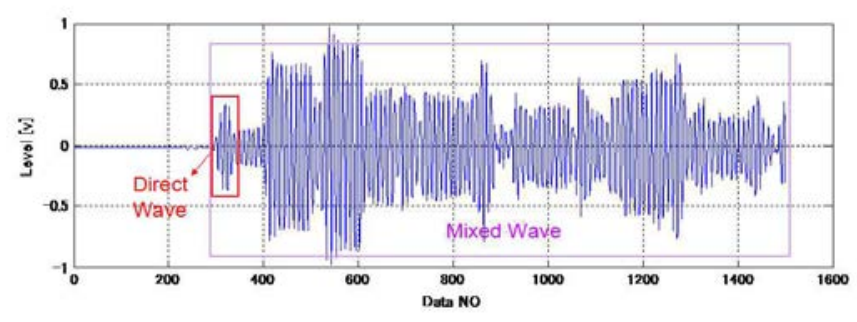

Fig. 3. A Example of Recevied Signal

Fig.3 shows an example of received signal. The first part of received signal is called "Direct Wave", which arrived at the reception transducer firstly. Then reflected waves arrive, and are mixed with direct waves. We call this part of wave "Mixed Wave”, which contains both direct waves and reflected waves.

Following with principle for measurement, the level of direct wave should be measured to calculate distance. We call this method "D_SPL". To measure the received voltage $V_{R}$, we need enough data to use in FFT. However, the received signal contains both direct and reflected waves, it's difficult to separate the direct wave in the received signal over long distance. Then there are no enough data to complete FFT accurately. 
To solve this problem, we paid attention to the mixed wave and reflected wave, and we found that the average level of mixed waves of some different frequencies is same as that of direct wave. Therefore, we use multiple-frequency sound to obtain the received voltage instead of a single-frequency sound. Using these data, we can measure distance for underwater robot in anyway. We call this new distance measuring method "M_SPL". In order to confirm the availability and performance of M_SPL, we performed many simulations.

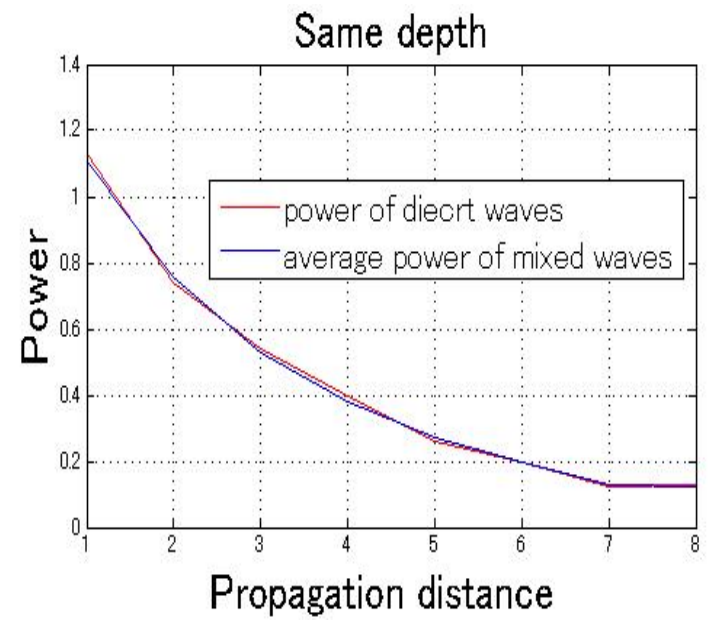

Fig. 4. Comparison of Power for Direct Wave and Mixed Wave

Fig.4 shows the results of a simulation to the change of the propagation distance. Here, we used from $32 \mathrm{kHz}$ to $40 \mathrm{kHz}$ as sound source. Using M_SPL, we measure the average level of mixed wave for them, and also measure the level of direct wave. Both results show that the level of direct wave and the average level of mixed wave are almost equal for every propagation distance.

We also carried out some experiments in the water-tank $(10[\mathrm{~m}] \times 50[\mathrm{~m}] \times 2[\mathrm{~m}])$ in Tokyo University of Marine Science and Technology (TUMSAT). We used the signal composed with multiple frequencies from $32 \mathrm{kHz}$ to $40 \mathrm{kHz}$ as sound source, and calculate distance using M_SPL.

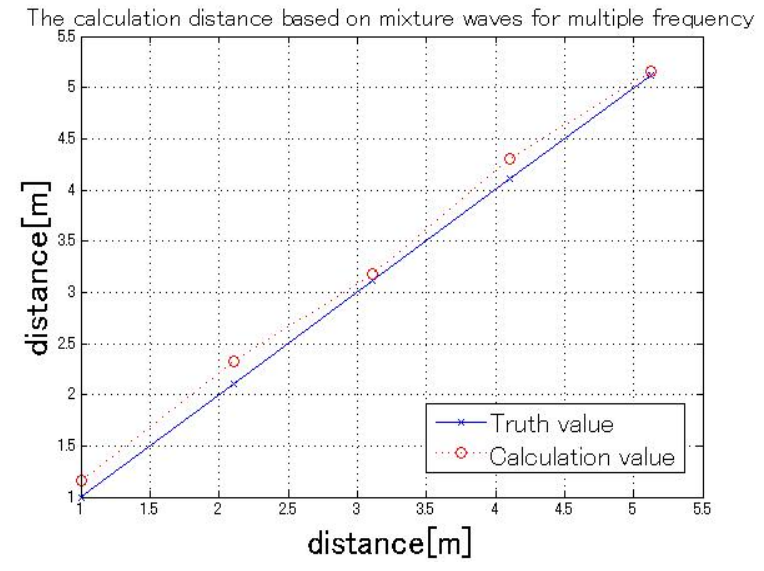

Fig. 5. Results of Distance
Fig.5 shows that the calculated distance from the average level of mixed wave as received voltage with using M_SPL. According to the results, we can see that M_SPL has higher accuracy and can be used availably.

\section{B. Position measutement based on sensor network}

On the basis of distance measurement, we propose positioning method with sensor network. We assume that each standard station is set of which distance from one to another is $200[\mathrm{~m}]$ to construct such a network.

Using the calculated distances $\left(R_{i}, i=1\right.$ to $\left.n\right)$ and positions $\left(x_{i}, y_{i}, z_{i}, i=1\right.$ to $\left.n\right)$ of each standard station from GPS, we can get an observation equation about position ( $\mathrm{x}, \mathrm{y}, \mathrm{z})$ of underwater robot which can be presented as

$$
R_{i}^{2}=\sqrt{\left(x_{i}-x\right)^{2}+\left(y_{i}-y\right)^{2}+\left(z_{i}-z\right)^{2}}
$$

Here, if receiving station is made as a center, measurement distance made as radius, the measurement sphere can be built. As an ideal case when every sphere meet at one point, it is the position of robot. However, there exist various error in every measured distance. So it is impossible that the measurement spheres meet at one point and the observation equation (3) cannot be solved. We need to make error correction.

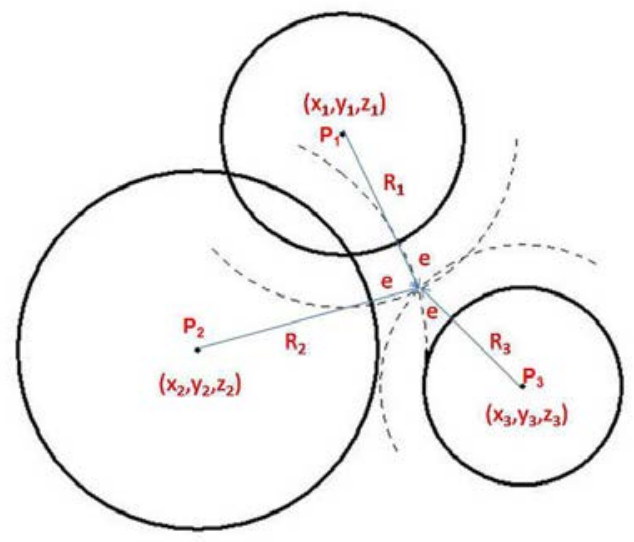

Fig. 6. Error Correction

Fig. 6 shows the process of error correction what make the spheres have the same one intersection by exchanging error with e. Then we can get a new observation equation about which is presented as

$$
R_{i}^{2}=\sqrt{\left(x_{i}-x\right)^{2}+\left(y_{i}-y\right)^{2}+\left(z_{i}-z\right)^{2}}+e
$$

We use the Newton's Method and the Least Squares Method to solve this observation equation. The Newton's method is used to modulate the error e repeatedly until the solution can be found. The Least Squares Method is used to solve the error problem when the number of observation equation is over. 
This position calculation method has already being used in many fields widely such as GSP. The availability also has been confirmed.

\section{PROTOTYPE SYSTEM}

In this section, we introduce the study of design and construction of prototype system for practical use.

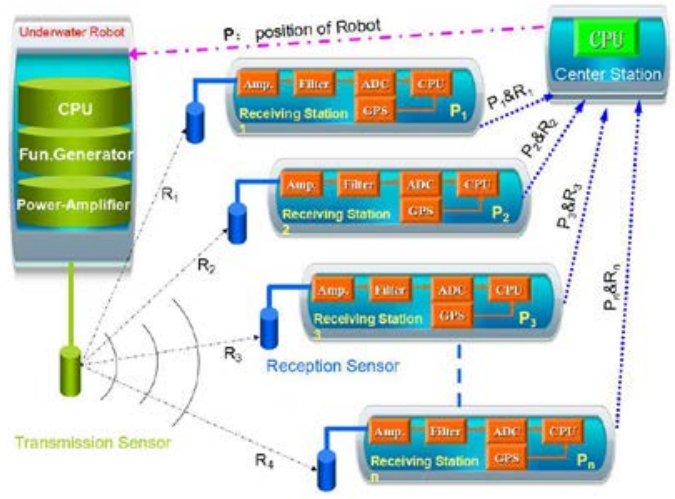

Fig. 7. Block Diagram of Prototype System

Fig.7 shows the block diagram of prototype system. The system contains transmission unit, reception unit and center unit. The transmission unit consists of Function.generator, Power-amplifier and Transducer, and it is equipped on underwater robot as sound source. The reception unit consists of Preamplifier, Filter, A/DC, Computing CPU and GPS. GPS takes charge of measuring position of reception unit, and CPU takes charge of computing of distance measurement. It is also used to send the distance and position data to the center unit. In center unit, CPU takes charge of receiving data from every reception unit by wireless communication, and calculating position of robot with using those data. It is also used to send the position data back to underwater robot for supporting its motion control.

\section{A. Transmission Unit}

In the part of distance measurement, we proposed a new method M_SPL. Because of its special function, there exist no facilities which can be respond to the M_SPL. So we developed a new Function.generator in transmission unit. For the M_SPL, transmission unit needs to generate the signal of multiple frequencies wave as sound source. Fig.8 shows the block circuit of Function.generator.

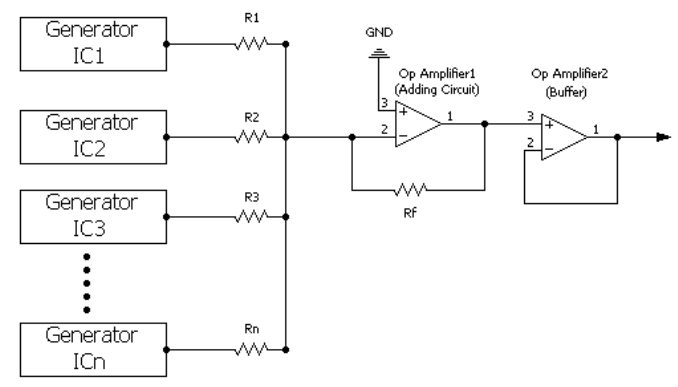

Fig. 8. Block Circuit of Fun.generatot
Here, we used some IC Wave Generators which can generate single frequency wave, and two operational amplifier (OP.amplifier) to complete the Function.generator. The first OP.amplifier is used as adding circuit to compound the waves from every IC. Another one is used for a buffer which can make the composed signal stable.

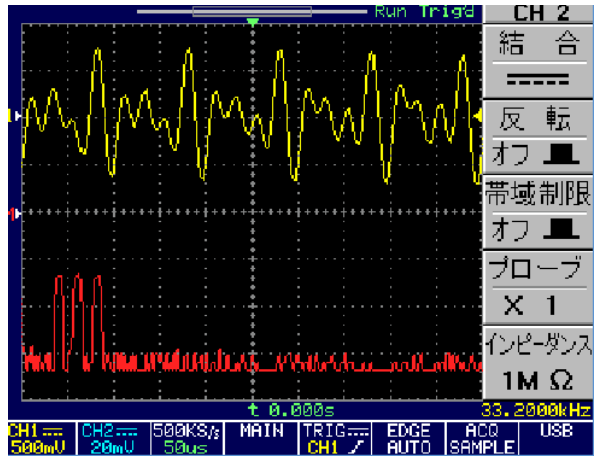

Fig. 9. A Test Result of composition Signal with Three Frequecies

Fig.9 shows a test result of composed signal from the new Function.generator with three frequencies. The top one shows the composed signal, and the bottom one shows the FFT result of the composed signal. It is clear that every frequency wave can be separated from composed signal. Therefore, the new Function.generator can be used as sound source availably in this system.

As sound source, the signal should be not continuous wave, but tone burst signal. Therefore, after the Function.generator, we use a switching circuit to control ON/OFF of the composed signal.

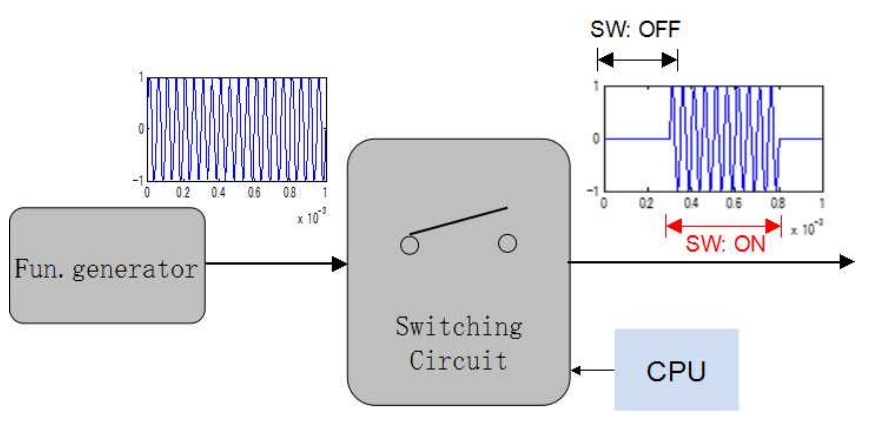

Fig. 10. Tone Burst Signal

Fig.10 shows the generator of tone burst signal with using switching circuit. The analog switch used in circuit is exchanged ON/OFF by CPU. When SW is ON, the continuous composed signal can be output, when SW is OFF, the signal can be not output. Thus the tone burst signal can be generated. The burst cycle and burst length can be controlled or set with CPU which used voltage signal to control the ON/OFF timing of SW.

Another important part of transmission unit is Poweramplifier. It consists of two parts of DC power and amplifier circuit. Using power supply transformer to exchange the level, and rectifier diode to change AC power to DC power. Then an OP.amplifier amplify the level of signal. The gain can be set optionally with using variable resistors. 
TABLE I. SPECIFICATION FOR TRANSMISSION UNIT

\begin{tabular}{|c|c|c|c|c|}
\hline Frequency & $\begin{array}{c}\text { Frequency } \\
\text { modulation }\end{array}$ & $\begin{array}{c}\text { Burst } \\
\text { Cycle }\end{array}$ & $\begin{array}{c}\text { Burst } \\
\text { Length }\end{array}$ & $\begin{array}{c}\text { Max } \\
\text { Output }\end{array}$ \\
\hline $\begin{array}{c}1 \sim 100 \\
{[\mathrm{kHz}]}\end{array}$ & Any & $1[\mathrm{~ms}] \sim$ & Any & $\begin{array}{c} \pm 100 \\
{[\mathrm{~V}]}\end{array}$ \\
\hline $\begin{array}{c}\text { Output } \\
\text { Offset }\end{array}$ & $\begin{array}{c}\text { Error } \\
\text { Rate }\end{array}$ & Size & Cost & Weight \\
\hline $\begin{array}{c} \pm 10 \\
{[\mathrm{mV}]}\end{array}$ & $2 \% \sim 3 \%$ & $\begin{array}{c}30 * 25 * 20 \\
{[\mathrm{~cm}]}\end{array}$ & $\begin{array}{c}80,000 \\
{[\mathrm{JPY}]}\end{array}$ & $\begin{array}{c}2.5 \\
{[\mathrm{~kg}]}\end{array}$ \\
\hline
\end{tabular}

The table shows the specification for complete transmission unit. This unit can generate the signal composed signal with any frequencies from $1 \mathrm{kHz} 100 \mathrm{kHz}$, and get the factors of tone burst signal optionally. The unit are completed with very small size and light weight. The cost is also cheap because it uses not special IC parts.

\section{B. Recption Unit}

The parts of Preamplifier, Filter, D/AC and Computing $\mathrm{CPU}$ in the reception unit are used for receiving the signal, and signal processing to calculate distance.

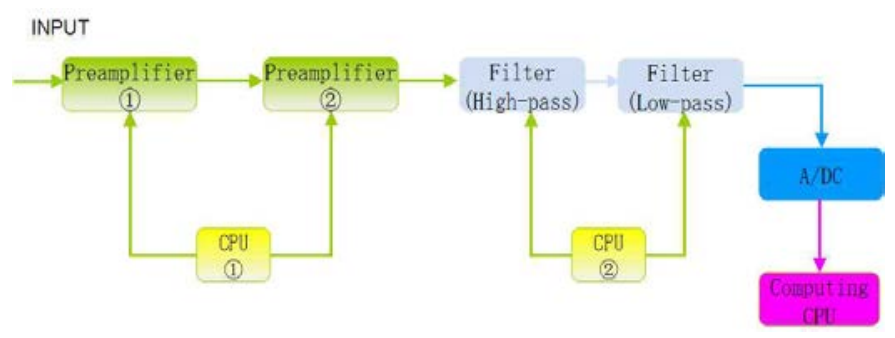

Fig. 11. Proess of Distance Calculation in Recption Unit

We used IC parts on the market to construct reception unit. Here, we use two preamplifiers to save enough large gain. Since we use multiple frequencies, we used one high-pass filter and one low-pass filter to make band-pass filter to cut noise. In order to calculate distance automatically, the amplifier gain and cutoff frequency for filter were set and changed in data processing with CPU. Then the signal of higher SN ratio can be recorded into computing CPU through D/AC. Distance will be calculated in CPU using these recorded data. The specification for reception unit is shown in the next table.

TABLE II. SPECIFICATION FOR RECEPTION UNIT

\begin{tabular}{|c|c|c|c|c|}
\hline Frequency & $\begin{array}{c}\text { Frequency } \\
\text { modulation }\end{array}$ & $\begin{array}{c}\text { Max } \\
\text { Gain }\end{array}$ & $\begin{array}{c}\text { Input } \\
\text { Loading Factor }\end{array}$ & $\begin{array}{c}\text { Max } \\
\text { Output }\end{array}$ \\
\hline $\begin{array}{c}1 \sim 100 \\
{[\mathrm{kHz}]}\end{array}$ & Any & $\times 10,000$ & $\begin{array}{c}100 \\
{[\mathrm{k} \Omega]}\end{array}$ & $\begin{array}{c} \pm 10 \\
{[\mathrm{~V}]}\end{array}$ \\
\hline $\begin{array}{c}\text { Output } \\
\text { Offset }\end{array}$ & $\begin{array}{c}\text { Error } \\
\text { Rate }\end{array}$ & $\begin{array}{c}\text { Attenuation } \\
\text { Slope }\end{array}$ & Cost & Weight \\
\hline $\begin{array}{c} \pm 20 \\
{[\mathrm{mV}]}\end{array}$ & $2 \% \sim 3 \%$ & $\begin{array}{c}12 \\
{[\mathrm{~dB} / \mathrm{oct}]}\end{array}$ & $\begin{array}{c}50,000 \\
{[\mathrm{JPY}]}\end{array}$ & $\begin{array}{c}1 \\
{[\mathrm{~kg}]}\end{array}$ \\
\hline
\end{tabular}

The other parts of reception unit are GPS equipment and CPU for wireless communication. GPS is used to measure position of receiving station, the CPU received the data and send them to center unit for calculating position of robot. For these equipment, we just used commercial products.

\section{Expriment}

To confirm the performance of transmission unit and reception unit, we carried out experiment in water tank. We used development unit and general-purpose measuring instrument to measure the average level of mixed wave under the same measuring condition. Fig.12 shows the comparison result between them.

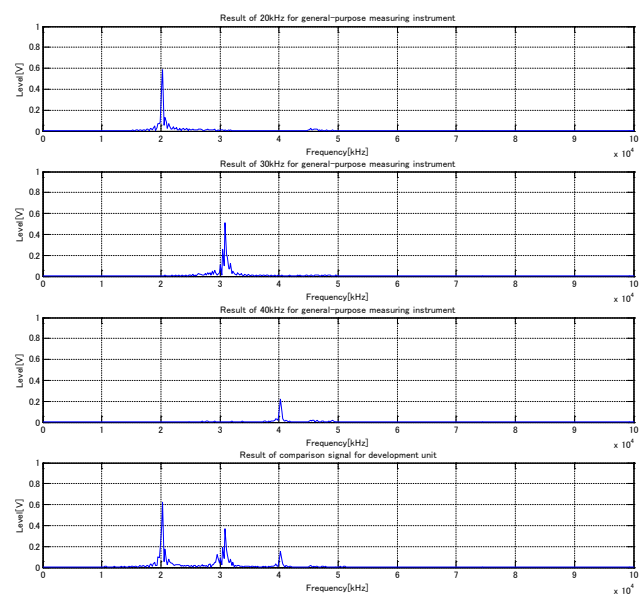

Fig. 12. Comparison Result of The Level of Mixed Wave

We used the signal generator for generational to generate single frequency signal of $20 \mathrm{kHz}, 30 \mathrm{kHz}$ and $40 \mathrm{kHz}$ as sound source, then measured the level of mixed wave from received signal for each one. The results is shown in top three graphs of Fig.12. We also generate the composed signal with $20 \mathrm{kHz}$, $30 \mathrm{kHz}$ and $40 \mathrm{kHz}$ as sound source. The result of mixed wave level is shown in bottom graph of Fig.12. Since the results are almost equal, it is clear that developed unit has the same performance with general-purpose measuring instrument, and can compound signal with any frequency. Therefore, these units can be used in positioning system availably.

\section{Center Unit}

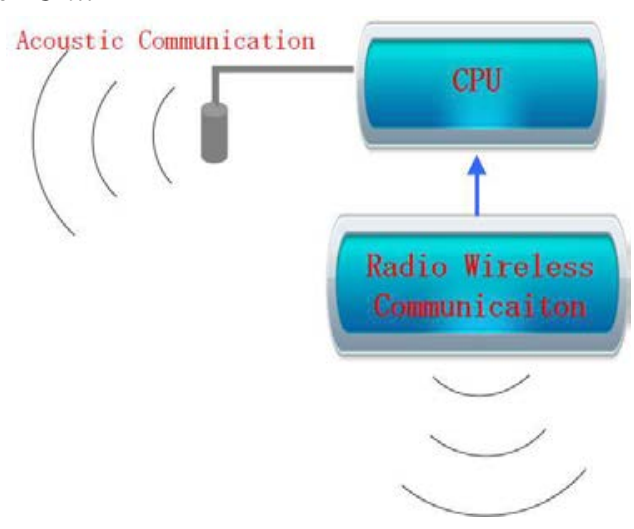

Fig. 13. Center Unit

The main composition of center unit is shown in Fig.13. The first part is for radio wireless communication. It takes charge of receiving the measured data from reception unit 
which is equipped on the float, and sending them to computing CPU. The other part is computing CPU. The computing CPU takes charge of calculating position of underwater robot using the data, and send the result to underwater robot to supporting its motion control with acoustic communication.

\section{COUNCLUSION}

In this study, we proposed a new positioning system based on sound propagation loss and sensor network.

- In the distance measurement, we proposed a new technology M_SPL which is using multiple frequencies wave for composed signal as sound source, and take the average level of their mixed waves from received signal, then use them as received voltage to calculate distance. The availability and performance of M_SPL was confirmed with simulations and experiments.

- In the position measurement, we proposed a method based on sensor network. Using the measured distance and data from sensor network, we can establish an observation equation with error correction. Then the position of robot can be calculated from this observation equation with the Newton's Method and the Least Squares Method.

- Based on the establishment of theory, we designed and constructed prototype system for practical use. In according to the originality of M_SPL. We used adding circuit to develop a new generator which can compound signal from multiple frequency waves as sound source of M_SPL. Then, we developed reception unit which can make data processing and calculate distance automatically. The availability and performance were also confirmed with verification of experiments.Merit

This system uses simple principle as the basic theory to measure distance, and measure position with using sensor network which has ad hoc and routing algorithm. This system has some merits as shown below.

- Since the simple basic principle, the data processing and computing become easy, and the measurement time is shortened.

- The position measurement is based on sensor network, the measuring range can be expanded when reception unit on float can be increased. And the accuracy and obstacle-proof performance can be improved with the sensor network.

- The system uses passive sonar which is based on single direction communication. There are only one transmission unit which is equipped on target and many reception units which are installed in a float and set on the sea surface. Therefore there does not exist mutual interference problem between units, and there is no necessity for mother ship companion. This system can be used for any kinds of robot which is just equipped in the transmission unit.

- The prototype system becomes simple, and can be developed with small size, cheap cost and light weight. Therefore, it is easy to use for whatever big or small robot.

This system has some merits, however there are some subjects which has not solved yet in present stage. In future, we will solve them as our next tasks.

- The first of next task is carrying out a final test of this system in open sea for the actual use of surveying sea floor with the underwater robot made by NAGASAKI UNIVERSITY in Japan, and improving the system.

- The error correction for measurement has not discussed yet in this study. It is also another importance task.

- M_SPL has proposed and used in this system. However the final proof of the physical phenomenon has not completed.

- When position was calculated, center unit should send the feedback data to underwater robot with acoustic communication. We also want to study about it.

- We should consider the possibility of the integrated use for this positioning system and conventional positioning systems.

\section{REFERENCES}

[1] X.Gao, F.Zhang, and M.Ito, "New Acoustic Positioning System for Underwater Robot Using Multiple Frequencies”, Journal of Artificial Life and Robotics, Vol.16, pp.524-545.

[2] X.Gao, F.Zhang, and M.Ito, "Underwater Acoustics Positioning System Based on Propagation Loss and Sensor Network," International conference on OCENAS 2012 KOREA.

[3] The Marine Acoutics Society of Japan, "Fundamental and Application of Marine Acoustics”, Seizando, 2004, pp.32-33.

[4] X.Gao, F.Zhang, and M.Ito,k.Mishima, "Motion Conrtrol of Underwater Robot with Positioning System Based on Sensor Network", The 82nd Scienntific Prsentatio, The Japan Institute of Marine Engineering (JIME), ISSN1346-1435, 2012, pp.53-54.

[5] X.Gao, "A New Positioning System Based on Multiple Frequencies", Master's Thesis, Tokyo University of Marine Science and Technology, Japan, Vol.1245, pp.21-32, 2011.

[6] Bin Fu, Feifei Zhang, Masanori Ito, "Development of a new positioning system for underwater robot based on sensor network", Journal of Artificial Life and Robotics, Vol.14, pp.284-289, 2009.

[7] Bin Fu, Feifei Zhang, Masanori Ito, "Development of a new underwater positioning system based on sensor network", Journal of Artificial Life and Robotics, Vol.13, pp.755-789, 2008.

[8] Y. Watanabe, H. Yoshida, H. Ochi, T. Hyakudome, Y. Nakano, S. Omika, M.Matuura, " Conceptual design of navigation of an AUV for Monitoring CCS site at deep sea bottom", Proc. of 30th International conference on Oceans, Offshore and Arctic Engineering, 2011.

[9] Hukusima, "GPS positioning system and program", Electronic Navigation Researcg Institute, pp.22 26, 2002. 